\title{
An Improved Diagonal Up-left Prediction Method for HEVC Intra Coding
}

\author{
Changnian Chen, Aiwu Shi ${ }^{+}$, Nan Su and E Chen \\ College of Mathematics and Computer, Cloud Computing \& Big Data Research Center, Wuhan Textile \\ University, Wuhan, China
}

\begin{abstract}
Intra prediction of HEVC supports up to 33 directional modes compared to 8 such modes in H.264/AVC. Employing many more prediction orientations, HEVC can reflect the directional properties of a coding picture more accurately. Although these directional modes are well designed and show good coding performance, the directional prediction strategy can still be improved. In this paper, an alternative diagonal up-left prediction mode is introduced. Two diagonal left reference samples are utilized to predict the mode with appropriate prediction weights. With negligible computing cost, the proposed prediction mode achieves better coding performance than the same directional mode of HEVC.
\end{abstract}

Keywords: intra coding, diagonal up-left prediction mode, HEVC.

\section{Introduction}

The High Efficiency Video Coding (HEVC) standard is developed jointly by the ITU-T Video Coding Experts Group (VCEG) and the ISO/IEC Moving Picture Experts Group (MPEG) standardization organizations [1]. Compared with its processor H.264/AVC, HEVC is supposed to have more coding efficiency and to be more suitable for high-resolution applications. Although the coding framework of HEVC is similar to that of H.264/AVC, one significant modification is the introduction of the quad-tree structure that is used in both intra and inter prediction coding. As for the intra prediction of HEVC, more prediction modes are supported for different prediction blocks with consistent prediction process [2].

Intra coding of HEVC achieves considerable coding gain while some efforts were made to put it forward. In [3], short distance intra prediction was proposed which split a coding unit into non-square units to shorten the distances between the predicted and the reference samples. Authors in [4] proposed a structured set prediction model that was based on a max-margin Markov network to regulate and optimize multiple block predictions. Kim et al. [5] introduced an improved DC prediction method for HEVC by pixel-by-pixel differential pulse code modulation. Fast mode selection is another research interests of intra coding [6,7].

In this paper, we focus on better directional prediction modes for HEVC intra coding. A new diagonal up-left prediction mode is asserted utilizing more reference samples with appropriate prediction weights. We first introduce the directional modes of HEVC standard briefly, and then our proposed scheme will be thoroughly illustrated and proved by experiments as well.

\section{Directional Modes of Intra Prediction}

To well support increased size of coding unit, intra prediction of HEVC supports a total of 33 prediction directions while H.264/AVC only supports 8 directional modes. These directional modes can be denoted as Intra_Angular[k], $2<\mathrm{k}<34$, as is shown in Fig. 1. Each directional mode represents a certain orientation. If we take these orientations in a semicircle, they have a fair angle between each of them. The dis-placement

\footnotetext{
+ Corresponding author. Tel.: + (027)59367297; fax: +(027)59367297.

E-mail address: cnccnu@163.com.
} 
differences become smaller when approaching vertical prediction mode (m26) and horizontal prediction mode (m10).

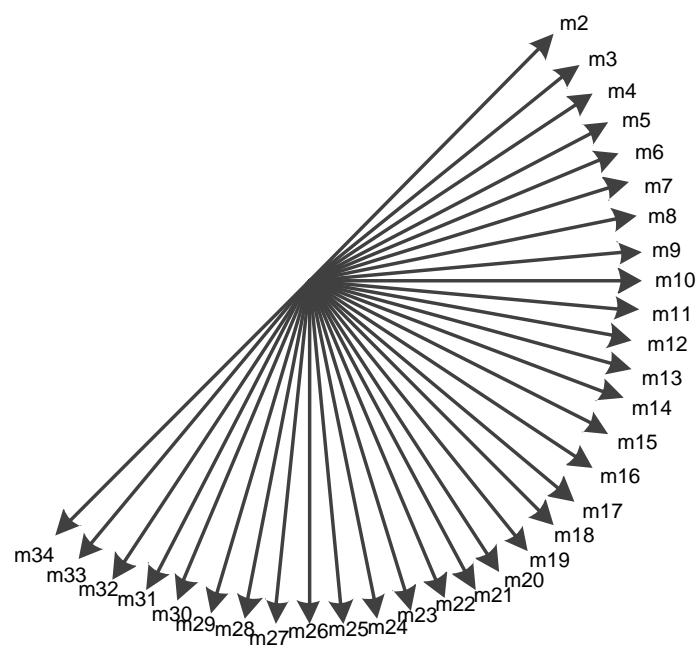

Fig. 1: Directional prediction modes of HEVC intra coding ( $\mathrm{m} 2$ to $\mathrm{m} 34$ ).

Prediction modes m26 and m10 still play an important role in HEVC as they do in H.264/AVC. However, the diagonal modes (m2, m18, and m34) turn out to be less important in HEVC than in H.264/AVC. These diagonal modes have similar probability to be the best mode as the rest directional modes. Since edge with diagonal information is still common in video sequences, this situation inspires us to find a more efficient way to represent these diagonal prediction modes.

\section{Proposed Method}

One improvement involved in this paper is for diagonal up-left mode $(\mathrm{m} 2)$. If we regard the directional modes as ones without arrows, it reveals that both $\mathrm{m} 2$ and $\mathrm{m} 34$ represent the diagonal left direction. Furthermore, example pixels referenced by these two modes can be utilized by both of them. As a consequence, we can improve one of the two modes with both references. As we known, the prediction process of the directional modes is consistent across all block sizes and prediction directions, and the upright reference samples are with more availability than left-down ones. It can be detected that double-pixel references are more effective for $\mathrm{m} 2$ than $\mathrm{m} 34$. Thus we improve $\mathrm{m} 2$ in the paper.

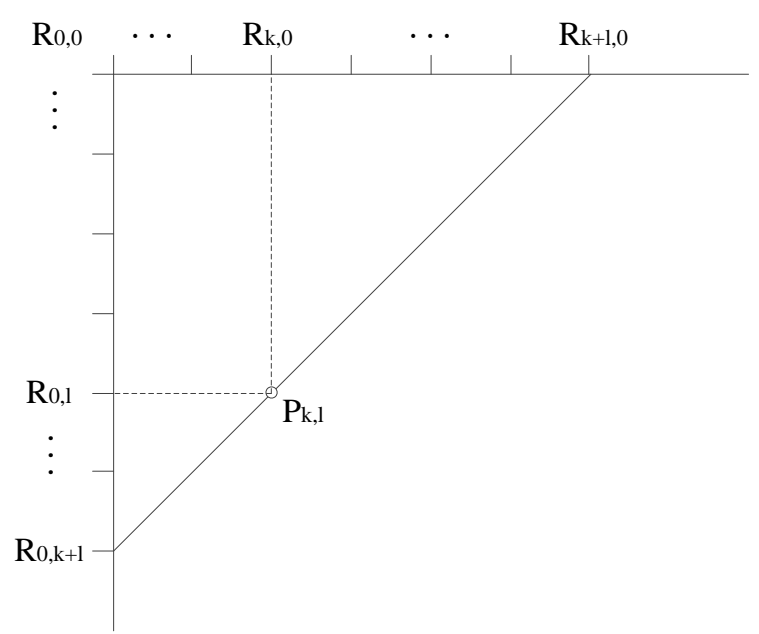

Fig. 2: Reference samples of directional mode m2.

According to the calculating formula of $\mathrm{m} 2$ in HEVC standard, the process can be very simple and consists of only copying integer reference samples from the reference row. In the proposed method, predicted sample is obtained by interpolating the reference samples in the diagonal left direction. For instance, predicted sample $P_{k, l}$ in Fig. 2 is calculated by reference samples $R_{0, k+l}$ and $R_{k+l, 0}$. Taking the 
mean of these two references is not a good option to reveal the spatial correlation between them and the predicted sample. Obviously, the reference nearer the predicted sample should take higher weight for it bears larger importance. Thus local correlation is taken into consideration when determining the weight of references. As proposed in [8], The weight is inversely proportional to the distance between the reference sample and the predicted sample. That is, if reference sample is near to predicted sample, it will have larger weight.

It is not difficult to find out that predicted sample $P_{k, l}$ of directional mode $\mathrm{m} 2$ is calculated as equation 1. Note that the slop of diagonal mode is 1 , and the proportion of the distance between $R_{0, k+l}$ and $P_{k, l}$ and that between $R_{k+l, 0}$ and $P_{k, l}$ is $l: k$.

$$
P_{k, l}=\frac{R_{k+l, 0} \times k+R_{0, k+l} \times l}{k+l}
$$

\section{Experimental Results}

To evaluate the performances of the proposed methods in this paper, the HM13.0 reference software is used under recommended test conditions [9]. Five video sequences of different resolutions (Traffic, ParkScene, RaceHorsesC, BlowingBubbles and Vidyo4) are tested. QPs are 22, 27, 32, and 37.

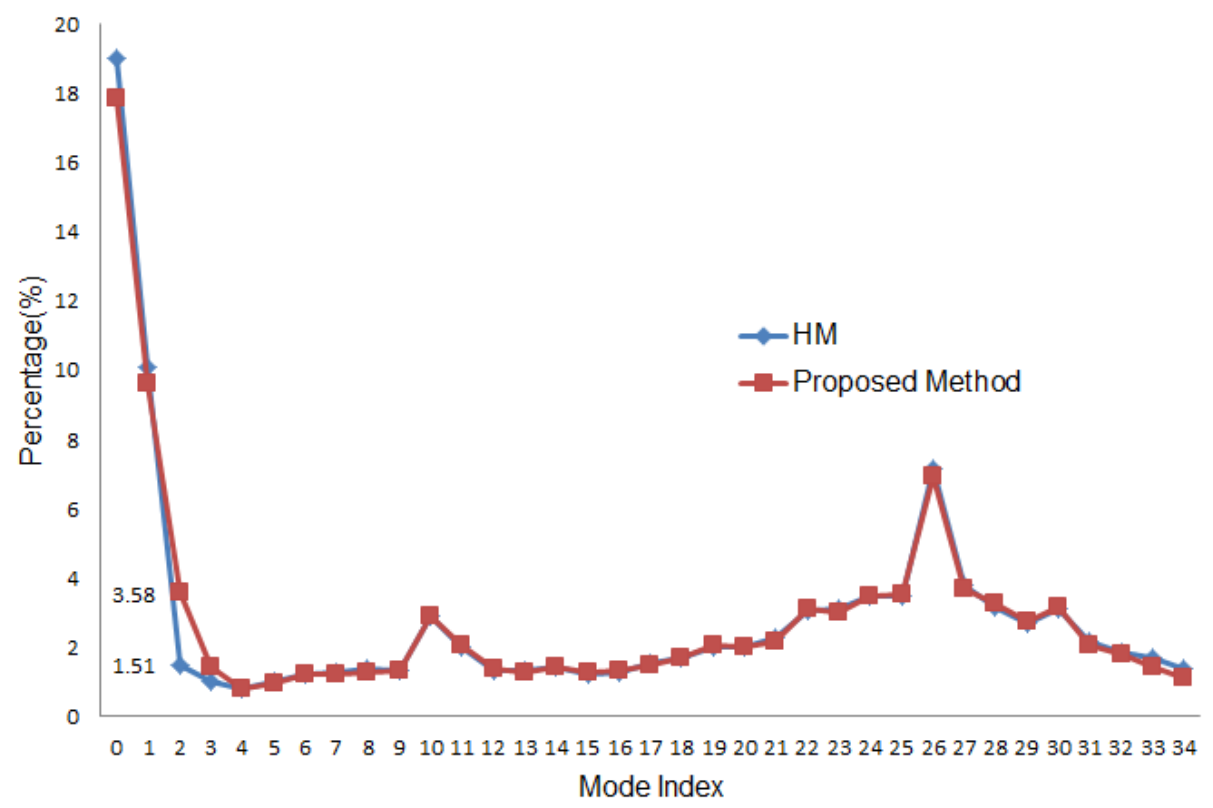

Fig. 3: Percentages of the prediction modes to be the best mode.

Table 1. Encoding Performance of the Proposed Method

\begin{tabular}{ccccccccc}
\hline \multirow{2}{*}{ Sequence } & \multicolumn{2}{c}{22} & \multicolumn{2}{c}{27} & \multicolumn{2}{c}{32} & \multicolumn{2}{c}{37} \\
\cline { 2 - 8 } & $\Delta$ PSNR & $\Delta \mathrm{BR}$ & $\Delta \mathrm{PSNR}$ & $\Delta \mathrm{BR}$ & $\Delta \mathrm{PSNR}$ & $\Delta \mathrm{BR}$ & $\Delta \mathrm{PSNR}$ & $\Delta \mathrm{BR}$ \\
\hline Class A & 0.004 & 0.09 & 0.002 & 0.07 & 0.008 & 0.07 & -0.001 & 0.13 \\
Class B & 0.017 & 0.01 & 0.003 & 0.05 & 0.002 & 0.11 & 0.002 & 0.13 \\
Class C & 0.011 & 0.06 & 0.004 & 0.04 & -0.004 & 0.201 & 0.003 & -0.09 \\
Class D & 0.005 & 0.09 & 0.012 & 0.03 & 0.001 & 0.11 & -0.001 & 0.10 \\
Class E & 0.004 & 0.03 & 0.002 & 0.03 & 0.003 & 0.15 & 0.003 & -0.05 \\
Average & 0.008 & 0.06 & 0.005 & 0.04 & 0.002 & 0.13 & 0.001 & 0.04 \\
\hline
\end{tabular}

Table 1 lays out results of the comparison between the proposed method and HM. The coding efficiency is measured by $\triangle \mathrm{PSNR}(\mathrm{dB})$ and $\triangle \mathrm{BR}(\%) . \triangle \mathrm{PSNR}$ represents the encoding gain and $\triangle \mathrm{BR}$ gives bitrate saving of the proposed method. The coding time increase is negligible so it is not considered. It illustrates 
that the proposed method is better than diagonal up-left mode with $0.004 \mathrm{~dB}$ coding gain and $0.07 \%$ bitrate saving in average. It also can see that our method is more efficient when QP set at a lower level. The reason of that is that encoding area is smoother with higher QP value and less directional mode will be selected to be the best mode. As a sequence, the benefit of adopting our method is limited.

\section{Conclusion}

In this paper, we propose an alternative method for diagonal up right mode of HEVC intra coding. Two reference samples along that orientation are employed for prediction. Their weights are determined by the distances between them and the predicted samples. Reference closer to the predicted sample has higher weight. Last but not least, our method can easily incorporate with other intra prediction methods.

\section{References}

[1] Sullivan, G.J., Ohm, J., Woo-Jin, H., Wiegand, T. Overview of the High Efficiency Video Coding (HEVC) Standard. IEEE Transactions on Circuits and Systems for Video Technology. 2012, 22, 1649-1668.

[2] Lainema, J., Bossen, F., Woo-Jin, H., Junghye, M., Ugur, K. Intra Coding of the HEVC Standard. IEEE Transactions on Circuits and Systems for Video Technology. 2012, 22, 1792-1801.

[3] Xiaoran, C., Changcai, L., Yunfei, W., Yun, H. Short distance intra coding scheme for HEVC. In: Picture Coding Symposium (PCS). 2012, 501-504.

[4] Wenrui, D., Hongkai, X., Xiaoqian, J., Chang Wen, C. Structured Set Intra Prediction with Discriminative Learning in a Max-Margin Markov Network for High Efficiency Video Coding. IEEE Transactions on Circuits and Systems for Video Technology. 2013, 23, 1941-1956.

[5] Kim, K., Jeon, G., Jeong, J.: Piecewise DC prediction in HEVC. Signal Processing: Image Communication. 2014.

[6] Siu, W.C., Wang, L.L.: Novel Adaptive Algorithm for Intra Prediction with Compromised Modes Skipping and Signaling Processes in HEVC. IEEE Transactions on Circuits and Systems for Video Technology. 2013.

[7] Chen, C., Chen, J., Xia, T., Ju, Z., Po, L.-M. An improved hybrid fast mode decision method for H.264/AVC intra coding with local information. Multimed Tools Appl. 2014, 72, 687-704.

[8] Yu, S.S., Gao, Y., Chen, J.Z., Zhou, J.L. Distance-based weighted prediction for H.264 Intra coding. In: International Conference on Audio, Language and Image Processing. 2008, 1, 1477-1480 1774.

[9] Bossen, F. Common test conditions and software reference configurations. Joint Collaborative Team on Video Coding (JCT-VC) of ITU-T VCEG and ISO/IEC MPEG, Geneva, CH, Doc.JCTVC-G1200, 2011. 